\title{
FAST REACTION LIMIT OF A VOLUME-SURFACE REACTION-DIFFUSION SYSTEM TOWARDS A HEAT EQUATION WITH DYNAMICAL BOUNDARY CONDITIONS
}

\author{
FELIX HENNEKE, BAO Q. TANG
}

\begin{abstract}
The fast reaction limit of a volume-surface reaction-diffusion system is rigorously investigated. The system is motivated by proteins localisation in stem cell division. By using Ball's energy equation method, we show that as the reaction rate constant goes to infinity, the solution of the original system converges to the solution of a heat equation with dynamical boundary condition. As a consequence, the dynamical boundary condition can be interpreted as a fast reaction limit of a volume-surface reaction-diffusion system.
\end{abstract}

\section{Introduction AND MAIN RESUlts}

Various physical phenomena in biology, material science, or chemical engineering are driven by reaction-diffusion processes in different compartments and by transfer between them. This may involve mass transfer between different domains but also with domain interfaces or boundaries. In cell-biology, for instance, many phenomena are based on reaction-diffusion processes of proteins within the cell cytoplasm and on the cell cortex [27,31]. Particular examples are systems modeling cell-biological signaling processes [19] or models for asymmetric stem cell division which describe the localisation of so-called cell-fate determinants during mitosis $[7,26,34]$.

In this paper, we investigate the asymptotic behaviour of the following volumesurface reversible chemical reaction

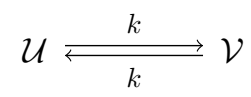

when the reaction rate constant $k \rightarrow+\infty$, where $\mathcal{U}$ is a volume-substance in $\Omega$ and $\mathcal{V}$ is a surface-substance on $\partial \Omega$. Here we assume that $\Omega \subset \mathbb{R}^{n}$ is a bounded domain with smooth boundary $\Gamma:=\partial \Omega$ (e.g. $\Gamma \in C^{2+\epsilon}$ for some $\epsilon>0$ ).

The study of (1.1) is motivated by models of proteins localisation in asymmetric stem cell division. In stem cells undergoing asymmetric cell division, particular proteins (so-called cell-fate determinants) are localised only on the cell cortex and have exchange reactions with proteins in the cell cytoplasm. For example, in SOP stem cells of Drosophila, the division operates around a key protein called Lgl (Lethal giant larvae) (see e,g. [7, 26, 34]). The reaction (1.1) thus can be viewed, for instance, as the exchange reaction between cytoplasmic Lgl and cortical Lgl with the reaction rate constant $k>0$. For a more complex system modelling the

2010 Mathematics Subject Classification. 35K57,35B4,35Q92.

Key words and phrases. Volume-surface reaction-diffusion systems; Fast reaction limit; Dynamical boundary condition; Energy equation method; Entropy method. 
localisation of Lgl, where the two different conformal states of Lgl are taken into account, the interested reader is referred to [18].

To set up a mathematical model for the reaction (1.1), we denote by $u(x, t)$ the volume-concentration of $\mathcal{U}$ and by $v(x, t)$ the surface-concentration of $\mathcal{V}$. The linear mass action volume-surface reaction-diffusion system modelling (1.1) reads as

$$
\begin{cases}u_{t}-d_{u} \Delta u=0, & x \in \Omega, \quad t>0, \\ d_{u} \partial_{\nu} u=-k(u-v), & x \in \Gamma, \quad t>0, \\ v_{t}-d_{v} \Delta_{\Gamma} v=k(u-v), & x \in \Gamma, \quad t>0, \\ u(x, 0)=u_{0}(x), & x \in \Omega, \\ v(x, 0)=v_{0}(x), & x \in \Gamma,\end{cases}
$$

where $\partial_{\nu}$ is the directional derivative corresponding to the unit outward normal vector $\nu$ of $\Gamma$, and $\Delta_{\Gamma}$ denotes the Laplace-Beltrami operator on $\Gamma$. Due to the reversibility of the reaction, the system (1.2) has the following conservation of the total mass

$$
\int_{\Omega} u(x, t) \mathrm{d} x+\int_{\Gamma} v(x, t) \mathrm{d} \sigma=\int_{\Omega} u_{0}(x) \mathrm{d} x+\int_{\Gamma} v_{0}(x) \mathrm{d} \sigma \quad \text { for all } \quad t>0 .
$$

Volume-surface reaction-diffusion (VSRD for short) systems have recently gained rapidly increasing attention as they occur naturally in many areas of applied mathematics as not only the mentioned cell biology but also as ecology, fluid dynamics or crystal growth, see e.g. $[1,6,4,5,15,19,22,28,25,30]$ and references therein.

On the other hand, fast reaction limits for reaction-diffusion systems have been increasingly active in recent years. In a reactive system, it frequently happens that certain reaction processes happen much faster than the other processes (e.g. diffusion processes or convection processes) and thus reach the (reaction) steady state quasi immediately. In such a case, the fast reactions can be eliminated to obtain a reduced system. The fast reaction limits of reactive systems occur commonly in chemical engineering and although applying such approximation has been routinely done by chemical engineers for a long time, the mathematical theory of fast reaction limits is usually missing. Several works have been carried out recently to rigorously prove fast reaction limit approximations (see e.g. $[8,9,10,18]$ and references therein).

In the present paper, we investigate a fast reaction limit for the VSRD system (1.2). We prove that, as the reaction rate constant $k \rightarrow+\infty$, the solution to (1.2) converge to the solution of a heat equation with a particular dynamical boundary condition (see e.g. [32]). As an interesting consequence, the dynamical boundary condition for the heat equation can be interpreted as the fast reaction limit of a volume-surface reaction-diffusion system; see [11, 20, 23, 24] for alternative derivations. Note that, up to the best of our knowledge, [18] is the only existing result concerning fast reaction limits for VSRD systems.

A problem similar to this work was studied in [9] where the authors proved the fast reaction limit for $\alpha \mathcal{U} \leftrightharpoons \beta \mathcal{V}$ with $\mathcal{U}$ and $\mathcal{V}$ are both volume-concentrations. We remark that because of the volume-surface coupling of (1.2), the technique used in [9] is not applicable here. This difficulty will be resolved in this paper by 
first applying famous Ball's energy equation technique (see e.g. [3]) to prove the fast reaction convergence in $L^{2}\left(0, T ; L^{2}(\Omega) \times L^{2}(\Gamma)\right)$, then exploiting the fact that system (1.2) and the limit equation (1.3) share the same equilibrium and both their solutions converge to this equilibrium, which is proved by entropy method, to show the fast reaction convergence in $L^{2}\left(0, T ; H^{1}(\Omega) \times H^{1}(\Gamma)\right)$. It is worth noticing that even though Ball's energy equation method is widely used in showing existence of attractors for PDEs, the present paper seems to be the first time the technique is used in a fast reaction limit problem.

Before stating our main results, let us define the notion of weak solution to a heat equation with dynamical boundary condition, which will be proved to be the limit of the system (1.2) as $k \rightarrow+\infty$.

Definition 1.1. For fixed $T>0$, a function $w \in C\left([0, T] ; L^{2}(\Omega)\right) \cap L^{2}\left(0, T ; H^{1}(\Omega)\right)$ is called a weak solution on $(0, T)$ to the heat equation with dynamical boundary condition

$$
\begin{cases}w_{t}-d_{u} \Delta w=0, & x \in \Omega, \quad t>0, \\ d_{u} \partial_{\nu} w=-w_{t}+d_{v} \Delta_{\Gamma} w, & x \in \Gamma, \quad t>0, \\ w(x, 0)=u_{0}(x), & x \in \Omega, \\ \left.w\right|_{\Gamma}(x, 0)=v_{0}(x), & x \in \Gamma,\end{cases}
$$

if there exists $z \in C\left([0, T] ; L^{2}(\Gamma)\right) \cap L^{2}\left(0, T ; H^{1}(\Gamma)\right)$ with $\left.w\right|_{\Gamma}(\cdot, t)=z(\cdot, t)$ almost everywhere in $(0, T)$ such that for all test functions $\varphi$ with $\left(\varphi,\left.\varphi\right|_{\Gamma}\right) \in C^{1}\left([0, T] ; H^{1}(\Omega) \times\right.$ $\left.H^{1}(\Gamma)\right)$ and $\varphi(T)=0$ we have

$$
\begin{aligned}
-\int_{0}^{T}\left\langle\left(w,\left.w\right|_{\Gamma}\right)\right. & \left.;\left(\varphi_{t},\left(\left.\varphi\right|_{\Gamma}\right)_{t}\right)\right\rangle \mathrm{d} t+d_{u} \int_{0}^{T} \int_{\Omega} \nabla w \nabla \varphi \mathrm{d} x \mathrm{~d} t \\
& +\left.\left.d_{v} \int_{0}^{T} \int_{\Gamma} \nabla_{\Gamma} w\right|_{\Gamma} \nabla_{\Gamma} \varphi\right|_{\Gamma} \mathrm{d} \sigma \mathrm{d} t=\left\langle\left(u_{0}, v_{0}\right) ;\left(\varphi(0),\left.\varphi\right|_{\Gamma}(0)\right)\right\rangle .
\end{aligned}
$$

Remark 1.1. Since $w$ has the trace $z=\left.w\right|_{\Gamma}$ belonging to $C\left([0, T] ; L^{2}(\Gamma)\right)$, the initial condition

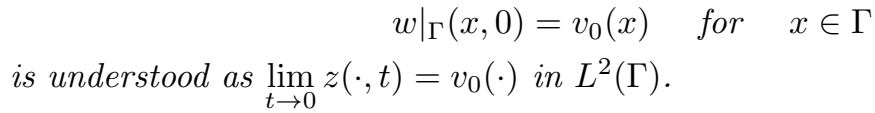

The main results of this paper are the following.

Theorem 1.1. Denote by $\left(u^{k}, v^{k}\right)$ the unique solution to system (1.2) subject to initial data $\left(u_{0}, v_{0}\right) \in L^{2}(\Omega) \times L^{2}(\Gamma)$ and reaction rate constant $k>0$. Then for $k \rightarrow+\infty$ there holds

$$
\left(u^{k}, v^{k}\right) \rightarrow\left(w,\left.w\right|_{\Gamma}\right) \quad \text { in } \quad L^{2}\left(0, T ; H^{1}(\Omega) \times H^{1}(\Gamma)\right),
$$

where $w$ is the unique weak solution to the heat equation with dynamical boundary condition (1.3).

Proof of Theorem 1.1. Here we sketch the proof of the Theorem based on some essential lemmas which will be proved in the next section.

By Lemma 2.3 we have $\left(u^{k}, v^{k}\right) \rightarrow(w, z)$ weakly in $L^{2}\left(0, T ; H^{1}(\Omega) \times H^{1}(\Gamma)\right)$ with $\left.w\right|_{\Gamma}=z$ and $w$ is a weak solution to the limit equation (1.3). Lemma 2.4 shows the strong convergence in $L^{2}\left(0, T ; L^{2}(\Omega) \times L^{2}(\Gamma)\right)$ of $\left(u^{k}, v^{k}\right) \rightarrow(w, z)$. Finally, by using 
Lemma 2.9, we obtain that $\left(u^{k}, v^{k}\right) \rightarrow(w, z)$ strongly in $L^{2}\left(0, T ; H^{1}(\Omega) \times H^{1}(\Gamma)\right)$. This completes the proof of the Theorem.

The remainder of this paper is structured as follows: In Section 2, we prove the main Theorem 1.1 by proving the Lemmas 2.3, 2.4 and 2.9 consecutively. We also briefly discuss a related nonlinear problem in the last Section 3.

\section{Proof of Theorem 1.1}

For the sake of brevity, throughout this paper, we denote by $\mathcal{L}^{2}=L^{2}(\Omega) \times L^{2}(\Gamma)$ and $\mathcal{H}^{1}=H^{1}(\Omega) \times H^{1}(\Gamma)$. The inner product in $\mathcal{L}^{2}$ is defined by

$$
\langle(u, v) ;(\varphi, \psi)\rangle:=\int_{\Omega} u \varphi \mathrm{d} x+\int_{\Gamma} v \psi \mathrm{d} \sigma
$$

which induces the norm $\|(u, v)\|_{\mathcal{L}^{2}}=\sqrt{\langle(u, v) ;(u, v)\rangle}$. For $t>0$, we denote by $\Omega_{t}=\Omega \times[0, t], \Gamma_{t}=\Gamma \times[0, t]$ and $L^{2}\left(\Omega_{t}\right)=L^{2}\left(0, t ; L^{2}(\Omega)\right), L^{2}\left(\Gamma_{t}\right)=L^{2}\left(0, t ; L^{2}(\Gamma)\right)$.

Since the domain $\Omega$ is assumed to be smooth enough, the boundary $\Gamma:=\partial \Omega$ is a smooth, compact Riemannian manifold without boundary with the natural metric inherited from $\mathbb{R}^{n}$, given in local coordinates by $\left(g_{i j}\right)_{i, j=1, \ldots, n-1}$. Hence we can define the Laplace-Beltrami operator on $\Gamma$ by

$$
\Delta_{\Gamma} u=g^{-1 / 2} \sum_{i, j=1}^{n-1} \frac{\partial}{\partial y_{i}}\left(g^{i j} g^{1 / 2} \frac{\partial u}{\partial y_{j}}\right)
$$

where $g=\operatorname{det}\left(g_{i j}\right)$ and $\left(g^{i j}\right)=\left(g_{i j}\right)^{-1}$ as usual. Throughout this paper, we will use the following identity

$$
\int_{\Gamma}\left(-\Delta_{\Gamma} u\right) v \mathrm{~d} \sigma=\int_{\Gamma} \nabla_{\Gamma} u \nabla_{\Gamma} v \mathrm{~d} \sigma
$$

where $\mathrm{d} \sigma$ is the natural volume element on $\Gamma$, with the local coordinates given by $\sqrt{g} d y_{1} \ldots d y_{n-1}$, and $\nabla_{\Gamma}$ is the Riemannian gradient. For more details of the Laplace-Beltrami operator, we refer the interested reader to [21].

2.1. Well Posedness and Limiting System. In this section we prove the existence results for the system (1.2) and the limiting equation (1.3). We also establish the weak convergence of solutions of (1.2) to solutions of (1.3) when $k \rightarrow \infty$.

Definition 2.1. For a fixed $T>0$, a pair of functions $(u, v) \in C\left([0, T] ; \mathcal{L}^{2}\right) \cap$ $L^{2}\left(0, T ; \mathcal{H}^{1}\right)$ is called a weak solution to $(1.2)$ on $(0, T)$ if for all test function $(\varphi, \psi) \in C^{1}\left([0, T] ; \mathcal{H}^{1}\right)$ satisfying $\varphi(T)=\psi(T)=0$ we have

$$
-\int_{0}^{T}\left\langle(u, v) ;\left(\varphi_{t}, \psi_{t}\right)\right\rangle \mathrm{d} t+\int_{0}^{T} a(u, v ; \varphi, \psi) \mathrm{d} t=\left\langle\left(u_{0}, v_{0}\right) ;(\varphi(0), \psi(0))\right\rangle
$$

where

$$
a(u, v ; \varphi, \psi)=d_{u} \int_{\Omega} \nabla u \nabla \varphi \mathrm{d} x+d_{v} \int_{\Gamma} \nabla_{\Gamma} v \nabla_{\Gamma} \psi \mathrm{d} \sigma+k \int_{\Gamma}(u-v)(\varphi-\psi) \mathrm{d} \sigma .
$$

Proposition 2.1. For any $\left(u_{0}, v_{0}\right) \in \mathcal{L}^{2}$, the system (1.2) possesses a unique weak solution $(u, v)$ on $(0, T)$ for all $T>0$ in the sense of Definition 2.1. 
Proof. It follows from direct computations that the bilinear form $a: \mathcal{H}^{1} \times \mathcal{H}^{1} \rightarrow \mathbb{R}$ defined in (2.2) is continuous and satisfies a Gårding inequality

$$
a(u, v ; u, v) \geq \alpha\|(u, v)\|_{\mathcal{H}^{1}}^{2}-c\|(u, v)\|_{\mathcal{L}^{2}}^{2}
$$

for some $\alpha, c>0$. The existence of a weak solution $(u, v)$ to $(1.2)$ then follows from standard theory of linear parabolic problems (see e.g. [13, XVIII §3)]).

Similarly, we can show the existence of a unique weak solution to the limit equation (1.3). The proof of the following Proposition is hence omitted.

Proposition 2.2. For any $\left(u_{0}, v_{0}\right) \in \mathcal{L}^{2}$, the equation (1.3) possesses a unique weak solution $w$ on $(0, T)$ for all $T>0$.

The well-posedness of $(1.3)$ in $L^{p}(\Omega) \times L^{p}(\Gamma)$ with $1 \leq p<+\infty$ and $p \neq 2$ is more subtle. The interested reader is referred to $[2,12,16,29]$ and references therein.

Remark 2.1. Note that we do not assume compatibility of the initial data in the sense $\left.u_{0}\right|_{\Gamma}=v_{0}$ in the case that the former exists. Compatibility is typically not satisfied for the reaction-diffusion system (1.2) and it is not needed for the existence of weak solutions. Lack of compatibility might, however, results in a lower regularity of the solution to (1.3) because if it were an element of $C\left([0, T], H^{1}(\Omega)\right)$ then a compatibility condition would follow. For a case where a compatibility condition is used see e.g. [33].

For the rest of this work, we will denote by $\left(u^{k}, v^{k}\right)$ the unique weak solution to (1.2) corresponding to the reaction rate constant $k>0$. Thanks to (2.1), we have

$$
\begin{aligned}
\left\|\left(u^{k}(t), v^{k}(t)\right)\right\|_{\mathcal{L}^{2}}^{2} & +2 d_{u}\left\|\nabla u^{k}\right\|_{L^{2}\left(\Omega_{t}\right)}^{2} \\
& +2 d_{v}\left\|\nabla_{\Gamma} v^{k}\right\|_{L^{2}\left(\Gamma_{t}\right)}^{2}+2 k\left\|u^{k}-v^{k}\right\|_{L^{2}\left(\Gamma_{t}\right)}^{2}=\left\|\left(u_{0}, v_{0}\right)\right\|_{\mathcal{L}^{2}}^{2} .
\end{aligned}
$$

for all $t \in(0, T]$. The relation (2.3) gives us the following important a priori estimates

$$
\begin{aligned}
& \left\{u^{k}\right\}_{k>0} \text { is bounded in } L^{\infty}\left(0, T ; L^{2}(\Omega)\right) \cap L^{2}\left(0, T ; H^{1}(\Omega)\right), \\
& \left\{v^{k}\right\}_{k>0} \text { is bounded in } L^{\infty}\left(0, T ; L^{2}(\Gamma)\right) \cap L^{2}\left(0, T ; H^{1}(\Gamma)\right)
\end{aligned}
$$

and

$$
\left\|u^{k}-v^{k}\right\|_{L^{2}\left(\Gamma_{T}\right)}=O\left(k^{-1 / 2}\right) \text { as } k \rightarrow+\infty .
$$

Combining (2.4), (2.5) and (2.6) allows us to have the following weak convergence result.

Lemma 2.3. There exist a function $w \in L^{2}\left(0, T ; H^{1}(\Omega)\right)$ and a function $z \in$ $L^{2}\left(0, T ; H^{1}(\Gamma)\right)$ such that

$$
u^{k} \rightarrow w \text { weakly in } L^{2}\left(0, T ; H^{1}(\Omega)\right)
$$

and

$$
v^{k} \rightarrow z \text { weakly in } L^{2}\left(0, T ; H^{1}(\Gamma)\right)
$$

as $k \rightarrow+\infty$. Moreover, we have $\left.w\right|_{\Gamma}=z$ and $w$ is the unique weak solution to the heat equation with dynamical boundary condition (1.3). 
Proof. The existence of $(w, z)$ and (2.7) and (2.8) follow from (2.4) and (2.5). We now verify that $\left.w\right|_{\Gamma}=z$ almost everywhere and $w$ solves (1.3) in weak sense.

From (2.6) we have, as $k \rightarrow+\infty, u^{k}-v^{k} \rightarrow 0$ strongly in $L^{2}\left(\Gamma_{T}\right)$ thus $u^{k}-v^{k} \rightarrow 0$ weakly in $L^{2}\left(\Gamma_{T}\right)$. It follows that, for any $\xi \in L^{2}\left(\Gamma_{T}\right)$, we have

$$
\lim _{k \rightarrow+\infty}\left(u^{k}-v^{k}, \xi\right)_{L^{2}\left(\Gamma_{T}\right)}=0 .
$$

Since $v^{k} \rightarrow z$ weakly in $L^{2}\left(0, T ; H^{1}(\Gamma)\right)$, we have

$$
\left(v^{k}, \xi\right)_{L^{2}\left(\Gamma_{T}\right)} \rightarrow(z, \xi)_{L^{2}\left(\Gamma_{T}\right)} .
$$

On the other hand, $u^{k} \rightarrow w$ weakly in $L^{2}\left(0, T ; H^{1}(\Omega)\right)$ then thus, $\left.\left.u^{k}\right|_{\Gamma} \rightarrow w\right|_{\Gamma}$ weakly in $L^{2}\left(\Gamma_{T}\right)$ thanks to the Trace Theorem. Therefore,

$$
\left(u^{k}, \xi\right)_{L^{2}\left(\Gamma_{T}\right)} \rightarrow(w, \xi)_{L^{2}\left(\Gamma_{T}\right)} .
$$

Hence, it follows from $(2.9)$ that $(w-z, \xi)=0$ for all $\xi \in L^{2}\left(\Gamma_{T}\right)$, which means $\left.w\right|_{\Gamma}=z$ almost everywhere.

We will show that $w$ is the solution to equation (1.3) subject to initial data $\left(u_{0}, v_{0}\right)$. By choosing a test function $\varphi \in C^{1}\left([0, T] ; H^{1}(\Omega)\right)$ satisfying $\left.\varphi\right|_{\Gamma} \in$ $C^{1}\left([0, T] ; H^{1}(\Gamma)\right)$ and $\varphi(T)=0$, it follows from $(2.1)$ that

$$
-\int_{0}^{T}\left\langle\left(u^{k}, v^{k}\right) ;\left(\varphi_{t},\left(\left.\varphi\right|_{\Gamma}\right)_{t}\right)\right\rangle \mathrm{d} t+\int_{0}^{T} a\left(u^{k}, v^{k} ; \varphi,\left.\varphi\right|_{\Gamma}\right) \mathrm{d} t=\left\langle\left(u_{0}, v_{0}\right) ;\left(\varphi(0),\left.\varphi\right|_{\Gamma}(0)\right)\right\rangle
$$

or equivalently

$$
\begin{aligned}
-\int_{0}^{T}\left\langle\left(u^{k}, v^{k}\right) ;\right. & \left.\left(\varphi_{t},\left(\left.\varphi\right|_{\Gamma}\right)_{t}\right)\right\rangle \mathrm{d} t+d_{u} \int_{0}^{T} \int_{\Omega} \nabla u^{k} \nabla \varphi \mathrm{d} x \mathrm{~d} t \\
& +\left.d_{v} \int_{0}^{T} \int_{\Gamma} \nabla_{\Gamma} v^{k} \nabla_{\Gamma} \varphi\right|_{\Gamma} \mathrm{d} \sigma \mathrm{d} t=\left\langle\left(u_{0}, v_{0}\right) ;\left(\varphi(0),\left.\varphi\right|_{\Gamma}(0)\right)\right\rangle .
\end{aligned}
$$

Passing to the limit in (2.10) as $k \rightarrow+\infty$ and recalling that $u^{k} \rightarrow w$ weakly in $L^{2}\left(0, T ; H^{1}(\Omega)\right)$ and $\left.v^{k} \rightarrow w\right|_{\Gamma}$ weakly in $L^{2}\left(0, T ; H^{1}(\Gamma)\right)$, we obtain

$$
\begin{aligned}
-\int_{0}^{T}\left\langle\left(w,\left.w\right|_{\Gamma}\right) ;\left(\varphi_{t},\right.\right. & \left.\left.\left(\left.\varphi\right|_{\Gamma}\right)_{t}\right)\right\rangle \mathrm{d} t+d_{u} \int_{0}^{T} \int_{\Omega} \nabla w \nabla \varphi \mathrm{d} x \mathrm{~d} t \\
& +\left.\left.d_{v} \int_{0}^{T} \int_{\Gamma} \nabla_{\Gamma} w\right|_{\Gamma} \nabla_{\Gamma} \varphi\right|_{\Gamma} \mathrm{d} \sigma \mathrm{d} t=\left\langle\left(u_{0}, v_{0}\right) ;\left(\varphi(0),\left.\varphi\right|_{\Gamma}(0)\right)\right\rangle .
\end{aligned}
$$

This verifies that $w$ is the weak solution to the equation (1.3) and hence the proof of the lemma is completed.

2.2. Strong convergence in $L^{2}\left(0, T ; \mathcal{L}^{2}\right)$.

Lemma 2.4. For any $T>0$ we have

$$
\left(u^{k}, v^{k}\right) \rightarrow(w, z) \quad \text { strongly in } \quad L^{2}\left(0, T ; \mathcal{L}^{2}\right)
$$

as $k \rightarrow+\infty$, where $w$ and $z$ are defined in Lemma 2.3.

Proof. The proof of this Lemma makes use of the energy equation method (see [3]). From Lemma 2.3 we have $\left(u^{k}, v^{k}\right) \rightarrow(w, z)$ weakly in $L^{2}\left(0, T ; \mathcal{L}^{2}\right)$ then

$$
\liminf _{k \rightarrow+\infty}\left\|\left(u^{k}, v^{k}\right)\right\|_{L^{2}\left(0, T ; \mathcal{L}^{2}\right)}^{2} \geq\|(w, z)\|_{L^{2}\left(0, T ; \mathcal{L}^{2}\right)}^{2} .
$$


With the help of $(2.11)$, to show $\left(u^{k}, v^{k}\right) \rightarrow(w, z)$ strongly in $L^{2}\left(0, T ; \mathcal{L}^{2}\right)$ we only need to prove that

$$
\limsup _{k \rightarrow+\infty}\left\|\left(u^{k}, v^{k}\right)\right\|_{L^{2}\left(0, T ; \mathcal{L}^{2}\right)}^{2} \leq\|(w, z)\|_{L^{2}\left(0, T ; \mathcal{L}^{2}\right)}^{2}
$$

Note that, for any $0<t \leq T$,

$$
\liminf _{k \rightarrow+\infty}\left\|\nabla u^{k}\right\|_{L^{2}\left(\Omega_{t}\right)}^{2} \geq\|\nabla w\|_{L^{2}\left(\Omega_{t}\right)}^{2} \text { and } \liminf _{k \rightarrow+\infty}\left\|\nabla_{\Gamma} v^{k}\right\|_{L^{2}\left(\Gamma_{t}\right)}^{2} \geq\left\|\nabla_{\Gamma} z\right\|_{L^{2}\left(\Gamma_{t}\right)}^{2}
$$

thanks to the weak convergence (2.7) and (2.8). From (2.3), we have, for all $t \in$ $(0, T]$

$$
\left\|\left(u^{k}(t), v^{k}(t)\right)\right\|_{\mathcal{L}^{2}}^{2}+2 d_{u}\left\|\nabla u^{k}\right\|_{L^{2}\left(\Omega_{t}\right)}^{2}+2 d_{v}\left\|\nabla_{\Gamma} v^{k}\right\|_{\Gamma}^{2} \leq\left\|\left(u_{0}, v_{0}\right)\right\|_{\mathcal{L}^{2}}^{2}
$$

Hence,

$$
\begin{aligned}
& \limsup _{k \rightarrow+\infty}\left\|\left(u^{k}(t), v^{k}(t)\right)\right\|_{\mathcal{L}^{2}}^{2} \\
& \leq \limsup _{k \rightarrow+\infty}\left(-2 d_{u}\left\|\nabla u^{k}\right\|_{L^{2}\left(\Omega_{t}\right)}^{2}-2 d_{v}\left\|\nabla_{\Gamma} v^{k}\right\|_{L^{2}\left(\Gamma_{t}\right)}^{2}\right)+\left\|\left(u_{0}, v_{0}\right)\right\|_{\mathcal{L}^{2}}^{2} \\
& \leq-2 d_{u} \liminf _{k \rightarrow+\infty}\left\|\nabla u^{k}\right\|_{L^{2}\left(\Omega_{t}\right)}^{2}-2 d_{v} \liminf _{k \rightarrow+\infty}\left\|\nabla_{\Gamma} v^{k}\right\|_{L^{2}\left(\Gamma_{t}\right)}^{2}+\left\|\left(u_{0}, v_{0}\right)\right\|_{\mathcal{L}^{2}}^{2} \\
& \leq-2 d_{u}\|\nabla w\|_{L^{2}\left(\Omega_{t}\right)}^{2}-2 d_{v}\left\|\nabla_{\Gamma} z\right\|_{L^{2}\left(\Gamma_{t}\right)}^{2}+\left\|\left(u_{0}, v_{0}\right)\right\|_{\mathcal{L}^{2}}^{2} \\
& =\|(w(t), z(t))\|_{\mathcal{L}^{2}}^{2}
\end{aligned}
$$

for all $t \in(0, T]$. The last equality of $(2.13)$ is due to the fact that $w$ is the solution to (1.3) and $z=\left.w\right|_{\Gamma}$. By using Fatou's lemma and (2.13) we have

$$
\begin{aligned}
\limsup _{k \rightarrow+\infty}\left\|\left(u^{k}, v^{k}\right)\right\|_{L^{2}\left(0, T ; \mathcal{L}^{2}\right)}^{2} & \leq \int_{0}^{T} \limsup _{k \rightarrow+\infty}\left\|\left(u^{k}(t), v^{k}(t)\right)\right\|_{\mathcal{L}^{2}}^{2} \mathrm{~d} t \\
& \leq\|(w, z)\|_{L^{2}\left(0, T ; \mathcal{L}^{2}\right)}^{2}
\end{aligned}
$$

and therefore obtain the desired inequality (2.12).

2.3. Convergence in $L^{2}\left(0, T ; \mathcal{H}^{1}\right)$. In this subsection, we show that the convergence $(2.7)$ and $(2.8)$ are actually strong in the topology of $L^{2}\left(0, T ; \mathcal{H}^{1}\right)$. In order to do that, we first show that (1.2) and (1.3) share the same unique equilibrium (depending on the initial mass) and both trajectories of (1.2) and (1.3) converge exponentially to this equilibrium as $t \rightarrow+\infty$. This is done by the so-called entropy method (see e.g. [14]). Then combining these convergences to equilibrium with another energy equation method, we will be able to show that $\left(\left|\nabla u^{k}\right|,\left|\nabla_{\Gamma} v^{k}\right|\right) \rightarrow\left(|\nabla w|,\left|\nabla_{\Gamma} z\right|\right)$ strongly in $L^{2}\left(0, T ; \mathcal{L}^{2}\right)$, which combined with $\left(u^{k}, v^{k}\right) \rightarrow(w, z)$ strongly in $L^{2}\left(0, T ; \mathcal{L}^{2}\right)$ leads to the strong convergence $\left(u^{k}, v^{k}\right) \rightarrow(w, z)$ in $L^{2}\left(0, T ; \mathcal{H}^{1}\right)$.

\subsubsection{Convergence to equilibrium.}

Lemma 2.5. Denote by $M:=\int_{\Omega} u_{0}(x) \mathrm{d} x+\int_{\Gamma} v_{0}(x) \mathrm{d} \sigma$ the initial mass. Then (1.2) and (1.3) obey the conservations of mass

$$
\int_{\Omega} u^{k}(x, t) \mathrm{d} x+\int_{\Gamma} v^{k}(x, t) \mathrm{d} \sigma=\int_{\Omega} w(x, t) \mathrm{d} x+\int_{\Gamma} w(x, t) \mathrm{d} \sigma=M
$$


for all $t>0$. Moreover, (1.2) and (1.3) possess the same equilibrium $\left(u_{\infty}, v_{\infty}\right)$, which solves

$$
\left\{\begin{array}{l}
u_{\infty}=v_{\infty} \\
|\Omega| u_{\infty}+|\Gamma| v_{\infty}=M
\end{array}\right.
$$

Proof. The proof relies on direct computations, so we omit it.

We are now proving that the solutions of (1.2) and (1.3) converge exponentially fast to the equilibrium defined in Lemma 2.5 and the convergence is independent of the reaction rate. We will use the method so-called entropy method (see e.g. [14]). The basic idea of the entropy method consists of studying the large-time asymptotics of a dissipative PDE model by looking for a nonnegative Lyapunov functional $\mathcal{E}(f)$ and its nonnegative entropy dissipation

$$
\mathcal{D}(f)=-\frac{\mathrm{d}}{\mathrm{d} t} \mathcal{E}(f(t))
$$

along the flow of the PDE model. The entropy dissipation $\mathcal{D}(f)$ satisfies

$$
\mathcal{D}(f)=0 \text { and conservation laws Leftrightarrow } f=f_{\infty} .
$$

If we can show an entropy-entropy dissipation estimate of the form

$$
\mathcal{D}(f) \geq \Phi\left(\mathcal{E}(f)-\mathcal{E}\left(f_{\infty}\right)\right), \quad \Phi(x) \geq 0, \quad \Phi(x)=0 \text { Leftrightarrowx }=0,
$$

for some nonnegative function $\Phi$, and if $\Phi^{\prime}(0) \neq 0$, one can usually get exponential convergence of $f(t)$ toward $f_{\infty}$ with a rate, which can be explicitly estimated.

To apply the entropy method to (1.2), we consider the entropy functional

$$
\mathcal{E}(f, g)=\|(f, g)\|_{\mathcal{L}^{2}}^{2}
$$

and its entropy dissipation

$$
\mathcal{D}^{k}(f, g)=2 d_{u}\|\nabla f\|_{L^{2}(\Omega)}^{2}+2 d_{v}\left\|\nabla_{\Gamma} g\right\|_{L^{2}(\Gamma)}^{2}+2 k\|f-g\|_{L^{2}(\Gamma)}^{2}
$$

where $f: \Omega \rightarrow \mathbb{R}$ and $g: \Gamma \rightarrow \mathbb{R}$. Remark that, if $(f, g)$ is a solution to the system (1.2), then it follows from standard calculations that

$$
\mathcal{D}^{k}(f(t), g(t))=-\frac{\mathrm{d}}{\mathrm{d} t} \mathcal{E}(f(t), g(t)) \quad \text { for all } \quad t>0 .
$$

The following entropy-entropy dissipation estimate is the main tool in showing the convergence to equilibrium.

Lemma 2.6. For any $k_{0}>0$ there exists $\lambda_{0}>0$ such that for all $k \geq k_{0}$ and all measurable functions $f: \Omega \rightarrow \mathbb{R}$ and $g: \Gamma \rightarrow \mathbb{R}$ satisfying $\int_{\Omega} f \mathrm{~d} x+\int_{\Gamma} g \mathrm{~d} \sigma=0$ we have

$$
\mathcal{D}^{k}(f, g) \geq \lambda_{0} \mathcal{E}(f, g) .
$$

Proof. By using the notation $\bar{f}=\frac{1}{|\Omega|} \int_{\Omega} f(x) \mathrm{d} x$ and $\bar{g}=\frac{1}{|\Gamma|} \int_{\Gamma} g(x) \mathrm{d} \sigma$ for the spatial averages of $f$ and $g$, the assumption $\int_{\Omega} f \mathrm{~d} x+\int_{\Gamma} g \mathrm{~d} \sigma=0$ can be rewritten as

$$
|\Omega| \bar{f}+|\Gamma| \bar{g}=0
$$

First, we compute

$$
\begin{aligned}
\mathcal{E}(f, g)=\|(f, g)\|_{\mathcal{L}^{2}}^{2} & =\|(f-\bar{f}, g-\bar{g})\|_{\mathcal{L}^{2}}^{2}+\|(\bar{f}, \bar{g})\|_{\mathcal{L}^{2}}^{2} \\
& =\mathcal{E}(f-\bar{f}, g-\bar{g})+\mathcal{E}(\bar{f}, \bar{g}) .
\end{aligned}
$$


By using the Poincaré inequalities

$$
\|\nabla f\|_{L^{2}(\Omega)}^{2} \geq C_{P}(\Omega)\|f-\bar{f}\|_{L^{2}(\Omega)}^{2} \quad \text { and } \quad\left\|\nabla_{\Gamma} g\right\|_{L^{2}(\Gamma)}^{2} \geq C_{P}(\Gamma)\|g-\bar{g}\|_{L^{2}(\Gamma)}^{2}
$$

for functions in $H^{1}(\Omega)$ and $H^{1}(\Gamma)$, respectively, we have

$$
\begin{aligned}
\frac{1}{2} \mathcal{D}^{k}(f, g) & \geq d_{u}\|\nabla f\|_{L^{2}(\Omega)}^{2}+d_{v}\left\|\nabla_{\Gamma} g\right\|_{L^{2}(\Gamma)}^{2} \\
& \geq d_{u} C_{P}(\Omega)\|f-\bar{f}\|_{L^{2}(\Omega)}^{2}+d_{v} C_{P}(\Gamma)\|g-\bar{g}\|_{L^{2}(\Gamma)}^{2} \\
& \geq \min \left\{d_{u} C_{P}(\Omega), d_{v} C_{P}(\Gamma)\right\} \mathcal{E}(f-\bar{f}, g-\bar{g}) .
\end{aligned}
$$

On the other hand, by using the trace inequality $\|\nabla f\|_{L^{2}(\Omega)}^{2} \geq C_{T}(\Gamma)\|f-\bar{f}\|_{L^{2}(\Gamma)}^{2}$ for functions in $H^{1}(\Omega)$, we get

$$
\begin{aligned}
\frac{1}{2} \mathcal{D}^{k}(f, g) & \geq d_{u} C_{T}(\Gamma)\|f-\bar{f}\|_{L^{2}(\Gamma)}^{2}+2 k\|f-g\|_{L^{2}(\Gamma)}^{2} \\
& \geq \frac{1}{2} \min \left\{d_{u} C_{T}(\Gamma), 2 k_{0}\right\}\|\bar{f}-g\|_{L^{2}(\Gamma)}^{2} \\
& =\frac{1}{2} \min \left\{d_{u} C_{T}(\Gamma), 2 k_{0}\right\}\left(\|\bar{f}-\bar{g}\|_{L^{2}(\Gamma)}^{2}+\|\bar{g}-g\|_{L^{2}(\Gamma)}^{2}\right) \\
& \geq \frac{1}{2} \min \left\{d_{u} C_{T}(\Gamma), 2 k_{0}\right\}\|\bar{f}-\bar{g}\|_{L^{2}(\Gamma)}^{2} \\
& =\frac{1}{2} \min \left\{d_{u} C_{T}(\Gamma), 2 k_{0}\right\}\left(\frac{|\Gamma|}{|\Omega|}+1\right) \mathcal{E}(\bar{f}, \bar{g})
\end{aligned}
$$

where we used (2.16) for the last equality. Now, by combining (2.17), (2.18) and (2.19) it yields (2.15) with

$$
\lambda_{0}=\min \left\{d_{u} C_{P}(\Omega), d_{v} C_{P}(\Gamma), d_{u} C_{T}(\Gamma) / 2, k_{0}\right\} .
$$

Proposition 2.7. The solution $\left(u^{k}, v^{k}\right)$ to (1.2) obeys the following convergence to equilibrium

$$
\left\|\left(u^{k}(t), v^{k}(t)\right)-\left(u_{\infty}, v_{\infty}\right)\right\|_{\mathcal{L}^{2}}^{2} \leq \mathrm{e}^{-\lambda_{0} t}\left\|\left(u_{0}, v_{0}\right)-\left(u_{\infty}, v_{\infty}\right)\right\|_{\mathcal{L}^{2}}^{2}
$$

for all $t>0$, where $k \geq k_{0}>0$ and $\lambda_{0}$ is independent of $t$ and $k$.

Proof. By defining $f=u^{k}-u_{\infty}$ and $g=v^{k}-v_{\infty}$, we have

$$
\left\{\begin{array}{lll}
\partial_{t} f-d_{u} \Delta f=0, & x \in \Omega, & t>0, \\
d_{u} \partial_{\nu} f=-k(f-g), & x \in \Gamma, & t>0, \\
\partial_{t} g-d_{v} \Delta_{\Gamma} g=k(f-g), & x \in \Gamma, & t>0,
\end{array}\right.
$$

with initial data $f(x, 0)=u_{0}(x)-u_{\infty}$ and $g(x, 0)=v_{0}(x)-v_{\infty}$. We calculate that $f$ and $g$ satisfies the mass conservation, for all $t>0$,

$$
|\Omega| \bar{f}(t)+|\Gamma| \bar{g}(t)=|\Omega| \bar{f}(0)+|\Gamma| \bar{g}(0)=0 .
$$

Therefore, we can apply Lemma 2.6 to have

$$
\mathcal{D}^{k}(f, g) \geq \lambda_{0} \mathcal{E}(f, g)
$$

Note that, from (2.14) we have $\mathcal{D}^{k}(f, g)=-\frac{\mathrm{d}}{\mathrm{d} t} \mathcal{E}(f, g)$. It then follows from Gronwall's lemma that

$$
\mathcal{E}(f, g)(t) \leq \mathrm{e}^{-\lambda_{0} t} \mathcal{E}(f(0), g(0)) \quad \text { for all } t>0 .
$$

The proof of the lemma is complete since (2.21) is equivalent to (2.20). 
Proposition 2.8. The solution $u$ to (1.3) satisfies the following convergence to equilibrium

$$
\left\|w(t)-u_{\infty}\right\|_{\Omega}^{2}+\left\|\left.w\right|_{\Gamma}(t)-v_{\infty}\right\|_{\Gamma}^{2} \leq \mathrm{e}^{-\lambda_{1} t}\left(\left\|u_{0}-u_{\infty}\right\|_{\Omega}^{2}+\left\|v_{0}-v_{\infty}\right\|_{\Gamma}^{2}\right)
$$

for all $t>0$ where $\lambda_{1}>0$ is independent of $t$.

Proof. The proof is similar to Lemma 2.7 with slight modifications so we omit it.

2.3.2. Strong convergence in $L^{2}\left(0, T ; \mathcal{H}^{1}\right)$.

Lemma 2.9. As $k \rightarrow+\infty$ there holds

$$
\left(u^{k}, v^{k}\right) \rightarrow(w, z) \quad \text { strongly in } \quad L^{2}\left(0, T ; \mathcal{H}^{1}\right)
$$

for all $T>0$, where $w$ and $z$ are defined in Lemma 2.3.

Proof. We will first prove that

$$
\left(\left|\nabla u^{k}\right|,\left|\nabla_{\Gamma} v^{k}\right|\right) \rightarrow\left(|\nabla w|,\left|\nabla_{\Gamma} z\right|\right) \quad \text { in } \quad L^{2}\left([0,+\infty) ; \mathcal{L}^{2}\right) .
$$

From the energy equation (2.3), we have

$$
\left\|\left(u^{k}(t), v^{k}(t)\right)\right\|_{\mathcal{L}^{2}}^{2}+2 d_{u}\left\|\nabla u^{k}\right\|_{L^{2}\left(\Omega_{t}\right)}^{2}+2 d_{v}\left\|\nabla_{\Gamma} v^{k}\right\|_{L^{2}\left(\Gamma_{t}\right)}^{2} \leq\left\|\left(u_{0}, v_{0}\right)\right\|_{\mathcal{L}^{2}}^{2} .
$$

From the limit equation (1.3), we have

$$
\|(w(t), z(t))\|_{\mathcal{L}^{2}}^{2}+2 d_{u}\|\nabla w\|_{L^{2}\left(\Omega_{t}\right)}^{2}+2 d_{v}\left\|\nabla_{\Gamma} z\right\|_{L^{2}\left(\Gamma_{t}\right)}^{2}=\left\|\left(u_{0}, v_{0}\right)\right\|_{\mathcal{L}^{2}}^{2} .
$$

Combining (2.23) and (2.24) yields

$$
\begin{aligned}
& \left\|\left(u^{k}(t), v^{k}(t)\right)\right\|_{\mathcal{L}^{2}}^{2}+2 d_{u}\left\|\nabla u^{k}\right\|_{L^{2}\left(\Omega_{t}\right)}^{2}+2 d_{v}\left\|\nabla_{\Gamma} v^{k}\right\|_{L^{2}\left(\Gamma_{t}\right)}^{2} \\
& \leq\|(w(t), z(t))\|_{\mathcal{L}^{2}}^{2}+2 d_{u}\|\nabla w\|_{L^{2}\left(\Omega_{t}\right)}^{2}+2 d_{v}\left\|\nabla_{\Gamma} z\right\|_{L^{2}\left(\Gamma_{t}\right)}^{2} .
\end{aligned}
$$

Letting $k \rightarrow+\infty$ and $t \rightarrow+\infty$ in (2.25), and using $\left(u^{k}(t), v^{k}(t)\right) \rightarrow\left(u_{\infty}, v_{\infty}\right)$ independently of $k$ and $(w(t), z(t)) \rightarrow\left(u_{\infty}, v_{\infty}\right)$ as $t \rightarrow+\infty$ we get

$$
\begin{gathered}
\limsup _{k \rightarrow+\infty}\left(2 d_{u}\left\|\nabla u^{k}\right\|_{L^{2}([0,+\infty) \times \Omega)}^{2}+2 d_{v}\left\|\nabla_{\Gamma} v^{k}\right\|_{L^{2}([0,+\infty) \times \Gamma)}^{2}\right) \\
\leq 2 d_{u}\|\nabla w\|_{L^{2}([0,+\infty) \times \Omega)}^{2}+2 d_{v}\left\|\nabla_{\Gamma} z\right\|_{L^{2}([0,+\infty) \times \Gamma)}^{2}
\end{gathered}
$$

On the other hand, (2.23) implies that

$$
\left\{\left(\left|\nabla u^{k}\right|,\left|\nabla_{\Gamma} v^{k}\right|\right)\right\} \text { is bounded in } L^{2}\left([0,+\infty) ; \mathcal{L}^{2}\right),
$$

thus

$$
\left(\left|\nabla u^{k}\right|,\left|\nabla_{\Gamma} v^{k}\right|\right) \rightarrow\left(|\nabla w|,\left|\nabla_{\Gamma} z\right|\right) \quad \text { weakly in } L^{2}\left([0,+\infty) ; \mathcal{L}^{2}\right) .
$$

Therefore, we have

$$
\liminf _{k \rightarrow+\infty}\left\|\left(\left|\nabla u^{k}\right|,\left|\nabla_{\Gamma} v^{k}\right|\right)\right\|_{L^{2}\left([0,+\infty) ; \mathcal{L}^{2}\right)}^{2} \geq\left\|\left(|\nabla w|,\left|\nabla_{\Gamma} z\right|\right)\right\|_{L^{2}\left([0,+\infty) ; \mathcal{L}^{2}\right)}^{2},
$$

which, combined with (2.26), yields that

$$
\lim _{k \rightarrow+\infty}\left\|\left(\left|\nabla u^{k}\right|,\left|\nabla_{\Gamma} v^{k}\right|\right)\right\|_{L^{2}\left([0,+\infty) ; \mathcal{L}^{2}\right)}^{2}=\left\|\left(|\nabla w|,\left|\nabla_{\Gamma} z\right|\right)\right\|_{L^{2}\left([0,+\infty) ; \mathcal{L}^{2}\right)}^{2} .
$$

From (2.27) and (2.28), we get

$$
\left(\left|\nabla u^{k}\right|,\left|\nabla_{\Gamma} v^{k}\right|\right) \rightarrow\left(|\nabla w|,\left|\nabla_{\Gamma} z\right|\right) \quad \text { strongly in } L^{2}\left([0,+\infty) ; \mathcal{L}^{2}\right) .
$$


This strong convergence, together with $\left(u^{k}, v^{k}\right) \rightarrow(w, z)$ strongly in $L^{2}\left(0, T ; \mathcal{L}^{2}\right)$, gives us the main result

$$
\left(u^{k}, v^{k}\right) \rightarrow(w, z) \quad \text { strongly in } L^{2}\left(0, T ; \mathcal{H}^{1}\right)
$$

for all $T>0$.

\section{Discussion for a Nonlinear Problem}

As a final remark to this paper, we consider the reversible reaction

$$
\alpha \mathcal{U} \underset{k}{\stackrel{k}{\rightleftarrows}} \beta \mathcal{V}
$$

where $\mathcal{U}$ and $\mathcal{V}$ are volume- and surface-concentrations respectively, and the stoichiometric coefficients $\alpha, \beta$ are positive. The system considered in the present paper is thus a special case of this reaction when $\alpha=\beta=1$. By applying the mass action law, this reaction results the following nonlinear VSRD system

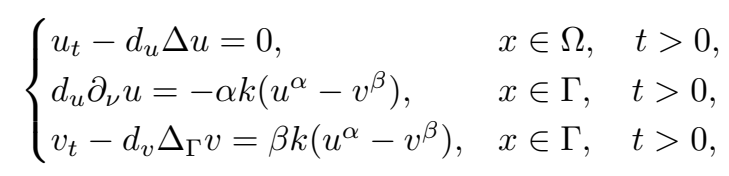

with suitable initial data. This system was proved to have a global weak solution which converges exponentially to equilibrium in [17].

Let the reaction rate constant $k$ tend to infinity, it is expected at least formally, that $\left(u^{k}, v^{k}\right) \rightarrow\left(w,\left.w\right|_{\Gamma}\right)$ where $w$ is a solution to the following heat equation with nonlinear dynamical boundary condition

$$
\begin{cases}w_{t}-d_{u} \Delta w=0, & x \in \Omega, \quad t>0 \\ d_{u} \partial_{\nu} w=\frac{\alpha}{\beta}\left(-\left(w^{\alpha / \beta}\right)_{t}+d_{v} \Delta_{\Gamma}\left(w^{\alpha / \beta}\right)\right), & x \in \Gamma, \quad t>0 \\ w(x, 0)=u_{0}(x), & x \in \Omega, \\ \left.w\right|_{\Gamma}(x, 0)=v_{0}(x)^{\alpha / \beta}, & x \in \Gamma .\end{cases}
$$

The analysis of this problem is much more involved compared to the linear case. Even the existence of solution to the limit equation with $\beta \neq \alpha$, up to the best of our knowledge, has not been shown in literature. The fast reaction limit problem for (3.1) remains as an interesting open problem.

Acknowledgements. The authors would like to thank the referee whose comment helps to improve the presentation of the paper.

A special thank goes to Prof. Fellner for his fruitful discussions. The authors are supported by International Research Training Group IGDK 1754.

\section{REFERENCES}

[1] B. Alberta, A. Johnson, J. Lewis, M. Raff, K. Roberts, and P. Walter. Molecular Biology of the Cell. 4th edn. New York: Garland Science (2002).

[2] Herbert Amann and Joachim Escher. Strongly continuous dual semigroups. Annali di Matematica Pura ed Applicata, 171(1):41-62, 1996.

[3] John M Ball. Global attractors for damped semilinear wave equations. Discrete and Continuous Dynamical Systems, 10(1/2):31-52, 2004.

[4] H. Berestycki, J.-M. Roquejoffre, and L. Rossi. Fisher-kpp propagation in the presence of a line: further effects. Nonlinearity, 26:2623-2640, 2013. 
[5] H. Berestycki, J.-M. Roquejoffre, and L. Rossi. The infulence of a line with fast diffusion on fisher-kpp propagation. J. Math. Biol., 66:743-766, 2013.

[6] Henri Berestycki, Anne-Charline Coulon, Jean-Michel Roquejoffre, and Luca Rossi. The effect of a line with nonlocal diffusion on fisher-kpp propagation. Mathematical Models and Methods in Applied Sciences, 25(13):2519-2562, 2015.

[7] Jörg Betschinger, Karl Mechtler, and Juergen A Knoblich. The par complex directs asymmetric cell division by phosphorylating the cytoskeletal protein lgl. Nature, 422(6929):326-330, 2003.

[8] D. Bothe. Instantaneous limits of reversible chemical reactions in presence of macroscopic convection. J. Differ. Equations, 193:27-48, 2003.

[9] D. Bothe and D. Hilhorst. A reaction-diffusion system with fast reversible reaction. J. Math. Anal. Appl., 286:125-135, 2003.

[10] D. Bothe and M. Pierre. The instantaneous limit for reaction-diffusion systems with a fast irreversible reaction. Discrete Contin. Dyn. Syst., 5:49-59, 2012.

[11] Xinfu Chen, Cody Pond, and Xuefeng Wang. Effective boundary conditions resulting from anisotropic and optimally aligned coatings: the two dimensional case. Archive for Rational Mechanics and Analysis, 206(3):911-951, 2012.

[12] Giuseppe Maria Coclite, Angelo Favini, Gisele Ruiz Goldstein, Jerome A Goldstein, and Silvia Romanelli. Continuous dependence on the boundary conditions for the wentzell laplacian. In Semigroup Forum, volume 77, pages 101-108. Springer, 2008.

[13] R. Dautray and J.-L. Lions. Mathematical Analysis and Numerical Methods for Science and Technology. Vol. 5. Springer-Verlag, Berlin, 1992.

[14] L. Desvillettes and K. Fellner. Exponential decay toward equilibrium via entropy methods for reaction-diffusion equations. J. Math. Anal. Appl., 319:157-176, 2006.

[15] C.M. Elliott and T. Ranner. Finite element analysis for a coupled bulk-surface partial differential equation. IMA J. Numer. Anal., 33:377-402, 2013.

[16] Angelo Favini, Gisèle Ruiz Goldstein, Jerome A Goldstein, and Silvia Romanelli. The heat equation with generalized wentzell boundary condition. Journal of Evolution Equations, $2(1): 1-19,2002$.

[17] K. Fellner, E. Latos, and B.Q. Tang. Well-posedness and exponential equilibration of a volume-surface reaction-diffusion system with nonlinear boundary coupling. arXiv:1404.2809, 2014.

[18] K. Fellner, S. Rosenberger, and B.Q. Tang. Quasi-steady-state approximation and numerical simulation for a volume-surface reaction-diffusion system. Comm. Math. Sci., (accepted) 2015.

[19] E. Friedmann, R. Neumann, and J.Rannacher. Well-posedness of a linear spatio-temporal model of the JAK2/STAT5 signaling pathway. Commun. Math. Anal., 15:76-102, 2013.

[20] G. R. Goldstein. Derivation and physical interpretation of general boundary conditions. Adv. Differential Equations, 11(4):457-480, 2006.

[21] J. Jost. Riemannian Geometry and Geometric Analysis, fifth ed., Universitext. SpringerVerlag, Berlin, 2008.

[22] Y.-I. Kwon and J. J. Derby. Modeling the coupled effects of interfacial and bulk phenomena during solution crystal growth. J. Cryst. Growth, 230:328-335, 2001.

[23] Huicong Li, Xuefeng Wang, and Yanxia Wu. The effective boundary conditions and their lifespan of the logistic diffusion equation on a coated body. Journal of Differential Equations, 257(10):3640-3668, 2014.

[24] Matthias Liero. Passing from bulk to bulk-surface evolution in the allen-cahn equation. NoDEA: Nonlinear Differential Equations and Applications, 20(3):919-942, 2013.

[25] Anotida Madzvamuse, Andy HW Chung, and Chandrasekhar Venkataraman. Stability analysis and simulations of coupled bulk-surface reaction-diffusion systems. In Proceedings of the Royal Society of London A: Mathematical, Physical and Engineering Sciences, volume 471, page 20140546. The Royal Society, 2015.

[26] Bernd Mayer, Gregory Emery, Daniela Berdnik, Frederik Wirtz-Peitz, and Juergen A Knoblich. Quantitative analysis of protein dynamics during asymmetric cell division. Current biology, 15(20):1847-1854, 2005.

[27] Ugo Mayor, Nicholas R Guydosh, Christopher M Johnson, J Günter Grossmann, Satoshi Sato, Gouri S Jas, Stefan MV Freund, Darwin OV Alonso, Valerie Daggett, and Alan R 
Fersht. The complete folding pathway of a protein from nanoseconds to microseconds. Nature, 421(6925):863-867, 2003.

[28] E. S. Medvedev and A. A. Stuchebrukhov. Proton diffusion along biological membranes. J. Phys. Condens. Matter, 23:234103, 2011.

[29] Delio Mugnolo. Vector-valued heat equations and networks with coupled dynamic boundary conditions. Advances in Differential Equations, 15(11/12):1125-1160, 2010.

[30] I. L. Novak, F. Gao, Y.-S. Choi, D. Resasco, J. C. Schaff, and B. M. Slepchenko. Diffusion on a curved surface coupled to diffusion in the volume: application to cell biology. J. Comput. Phys., 226:1271-1290, 2007.

[31] Igor L Novak, Fei Gao, Yung-Sze Choi, Diana Resasco, James C Schaff, and Boris M Slepchenko. Diffusion on a curved surface coupled to diffusion in the volume: application to cell biology. Journal of computational physics, 226(2):1271-1290, 2007.

[32] S. Romanelli. Goldstein-Wentzell boundary conditions: recent results with Jerry and Gisèle Goldstein. Discrete Contin. Dyn. Syst., 34(2):749-760, 2014.

[33] Juan Luis Vázquez and Enzo Vitillaro. Heat equation with dynamical boundary conditions of reactive-diffusive type. Journal of Differential Equations, 250(4):2143-2161, 2011.

[34] Frederik Wirtz-Peitz, Takashi Nishimura, and Juergen A Knoblich. Linking cell cycle to asymmetric division: Aurora-a phosphorylates the par complex to regulate numb localization. Cell, 135(1):161-173, 2008.

TANG QUOC BAO ${ }^{1,2}$

1 Institute of Mathematics and Scientific Computing, University of Graz, HeinrichStrasse 36, 8010 Graz, Austria

2 Faculty of Applied Mathematics and Informatics, Hanoi University of Sience and Technology, 1 Dai Co Viet, Hai Ba Trung, Hanoi, Vietnam

E-mail address: quoc.tang@uni-graz.at

Felix Henneke

Technische Universität München, Zentrum Mathematik, Boltzmannstrasse 3, 85748 GarchING, Germany

E-mail address: henneke@ma.tum.de 